\title{
All-fiber femtosecond Cherenkov radiation source
}

Liu, Xiaomin ; Lægsgaard, Jesper; Møller, Uffe; Tu, Haohua ; Boppart, Stephen A. ; Turchinovich, Dmitry

Published in:

Optics Letters

Link to article, DOI:

10.1364/OL.37.002769

Publication date:

2012

Document Version

Publisher's PDF, also known as Version of record

Link back to DTU Orbit

Citation (APA):

Liu, X., Lægsgaard, J., Møller, U., Tu, H., Boppart, S. A., \& Turchinovich, D. (2012). All-fiber femtosecond Cherenkov radiation source. Optics Letters, 37(13), 2769-2771. https://doi.org/10.1364/OL.37.002769

\section{General rights}

Copyright and moral rights for the publications made accessible in the public portal are retained by the authors and/or other copyright owners and it is a condition of accessing publications that users recognise and abide by the legal requirements associated with these rights.

- Users may download and print one copy of any publication from the public portal for the purpose of private study or research.

- You may not further distribute the material or use it for any profit-making activity or commercial gain

- You may freely distribute the URL identifying the publication in the public portal

If you believe that this document breaches copyright please contact us providing details, and we will remove access to the work immediately and investigate your claim. 


\title{
All-fiber femtosecond Cherenkov radiation source
}

\author{
Xiaomin Liu, ${ }^{1}$ Jesper Lægsgaard, ${ }^{1}$ Uffe Møller, ${ }^{1}$ Haohua Tu, ${ }^{2}$ Stephen A. Boppart, ${ }^{2}$ and Dmitry Turchinovich ${ }^{1,3, *}$ \\ ${ }^{1}$ DTU Fotonik, Technical University of Denmark, Lyngby DK-2800 Kgs., Denmark \\ ${ }^{2}$ Biophotonics Imaging Laboratory, University of Illinois at Urbana-Champaign, Urbana, Illinois 61801, USA \\ ${ }^{3}$ Max Planck Institute for Polymer Research, Ackermannweg 10, Mainz 55128, Germany \\ ${ }^{*}$ Corresponding author: dmtu@fotonik.dtu.dk
}

Received April 2, 2012; revised May 15, 2012; accepted May 15, 2012;

posted May 16, 2012 (Doc. ID 165995); published June 29, 2012

\begin{abstract}
An all-fiber femtosecond source of spectrally isolated Cherenkov radiation is reported, to the best of our knowledge, for the first time. Using a monolithic, self-starting femtosecond Yb-doped fiber laser as the pump source and the combination of photonic crystal fibers as the wave-conversion medium, we demonstrate milliwatt-level, stable, and tunable Cherenkov radiation at visible wavelengths $580-630 \mathrm{~nm}$, with pulse duration of sub-160-fs, and the $3 \mathrm{~dB}$ spectral bandwidth not exceeding $36 \mathrm{~nm}$. Such an all-fiber Cherenkov radiation source is promising for practical applications in biophotonics such as bioimaging and microscopy. (c) 2012 Optical Society of America

OCIS codes: $\quad 060.2310,060.5295,140.7090,320.2250,320.7110$.
\end{abstract}

Traditionally, Cherenkov radiation (CR) is known as light produced by charged particles passing through a given dielectric medium at speeds greater than the phase velocity of light in that medium [1]. In nonlinear fiber optics, the concept of $\mathrm{CR}$, also called dispersive wave generation or nonsolitonic radiation, originates from the perturbation of a stable temporal soliton by the higher-order fiber dispersion [2,3], which has a formal mathematical analogy to the traditional CR. This process is a key characteristic in the fiber-based supercontinuum (SC) sources that have been developed over the past decade []].

While picosecond-pumped fiber SC sources are of interest for the generation of high-brightness near-IR (NIR), visible (VIS), and UV light, they have limitations in terms of noise characteristics [5] and pulse duration [4]. For a femtosecond-pumped $\mathrm{S} \overline{\mathrm{C}}$, low average power

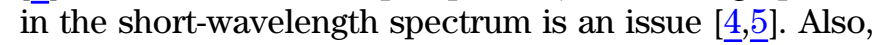
an all-fiber solution for a femtosecond SC source has not been demonstrated so far.

An attractive alternative to SC for ultrafast NIR, VIS, or UV generation in fiber-based systems is the generation of narrowband $\mathrm{CR}$ output in short pieces of photonic crystal fiber (PCF) using femtosecond pump pulses [ $[$ ] A milliwatt-level CR can be generated in this way, which is wavelength-tunable across the NIR-VIS-UV spectral range by choosing the PCFs with suitable zero-dispersion wavelength (ZDW). This fiber-optic-generated $\mathrm{CR}$ is attractive for multimodality biophotonics imaging, such as discussed in [7]. To the best of our knowledge, all previously demonstrated femtosecond CR fiber sources were based on systems with free-space optical coupling, using solid-state lasers as pumps. Solid-state lasers usually have a rather high pulse quality as compared to fiber lasers. On the other hand, the free-space optical coupling to thin-core PCFs used for CR naturally leads to environmental instability of such CR-generation systems and makes them not suitable for practical biophotonics imaging applications outside of the laboratory. Importantly for this work, CR generation in fibers is not critically dependent on the pump pulse duration since the input pulse is easily compressed in fibers. As will be shown below, the pulses from all-fiber laser systems, with spectro-temporal properties more complicated than of their solid-state counterparts, can generate sub-160-fs, relatively clean $\mathrm{CR}$ pulses. At the same time, such a monolithic, all-fiber CR-generation system provides a very high environmental and operational stability, defined by that of a self-stabilized all-fiber laser used as a pump.

In this Letter, we present an all-fiber, fully monolithic system based on an Yb-fiber femtosecond laser, producing electrically tunable femtosecond CR output in the spectral range of 580-630 nm with average power in the milliwatt range. Its layout is shown in Fig. 1 . The system consists of two parts: an all-fiber nanojoule-level selfstabilized [8] femtosecond laser operating at $1035 \mathrm{~nm}$ wavelength and $26.7 \mathrm{MHz}$ repetition rate used as the pump source, and a spliced-on multisegment PCF link used for Cherenkov wave conversion. More details of the pump laser can be found in $[\underline{9}, \underline{10}]$ and references therein. The small-core nonlinear P $\overline{\mathrm{C}}$ NL-3.0-850 (NKT Photonics $\mathrm{A} / \mathrm{S}$ ), which has a ZDW around $850 \mathrm{~nm}$, is used for $\mathrm{CR}$ generation. The mode-field diameters (MFDs) of the NL-3.0-850 fiber and the hollow-core anomalous dispersion PCF (HC-PCF) delivering the pulse-compressed pump laser signal are 1.8 and $7.5 \mu \mathrm{m}$, respectively. Therefore, the direct splicing of these two fibers would result in

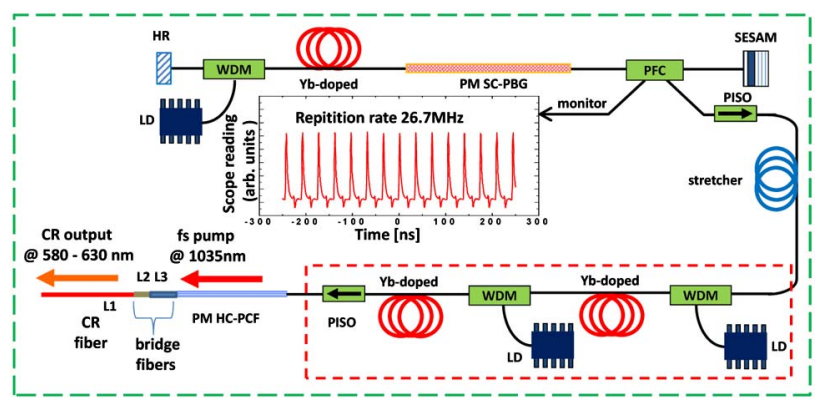

Fig. 1. (Color online) Layout of the all-fiber Cherenkov source. HR, high-reflectivity pigtailed mirror; WDM, 980/1030 wavelength division multiplexer; $\mathrm{LD}$, single-mode pumping diode at $974 \mathrm{~nm}$; PM SC-PBG, polarization-maintaining all-solid photonic bandgap fiber; SESAM, semiconductor saturable absorber mirror; PISO, polarization-maintaining isolator; PFC, 80/20 polarization filter coupler; HC-PCF, laser pulse compressor hollow-core PCF; CR fiber, highly nonlinear fiber for CR generation. (Inset) Oscilloscope reading of the mode-locked pulse train. 
an enormous coupling loss of around $12 \mathrm{~dB}$. In order to minimize this loss, two other types of fibers are spliced in between them as bridge fibers. The MFDs of the two bridge fibers are 3.3 and $6.6 \mu \mathrm{m}$ respectively, providing a gradual reduction of MFD in the fiber link. As a result, the total splicing loss in such a multisegment CR link is found to be around $4 \mathrm{~dB}$. The NL-3.0-850 fiber and the two bridge fibers are $10 \mathrm{~cm}(\mathrm{~L} 1), 2 \mathrm{~mm}$ (L2), and $8 \mathrm{~cm}$ (L3) long, respectively.

The $1035 \mathrm{~nm}$ pump pulses used in our experiment had an autocorrelation (AC) FWHM of 460 fs when fully compressed [9]. In this system, to maximize the CR output, the pump pulses were negatively prechirped with group delay dispersion of $-1.46 \cdot 10^{4} \mathrm{fs}^{2}$ in order to provide the shortest pulse duration and hence highest peak intensity while in the CR-generating NL-3.0-850 fiber. We note here that the dispersion of the bridge fibers used in the link is normal for the pump wavelength.

The pump pulses propagating in the small-core PCF initially undergo nonlinear pulse compression due to the combination of self-phase modulation (SPM) and anomalous dispersion. Around the point of maximal compression, the ultrafast VIS CR pulses are generated. Figure 2(a) shows the emitted CR output spectra measured at different output powers, and the inset shows the typical spectrum of the input pump pulse.

The CR phase-matching condition implies that the generated CR wavelength is dependent on the peak power of the pump pulse. In our experiments, when the $1035 \mathrm{~nm}$ pump power was increasing from 130 to $330 \mathrm{~mW}$, the central wavelength of the emitted CR was shifting from 630 to $580 \mathrm{~nm}$, yielding the blueshift as expected in this process [11]. This spectral shift is based on the control of the chirp and bandwidth of $1035 \mathrm{~nm}$ pump pulse by controlling the inversion in the amplifier section of the $\mathrm{Yb}$-fiber laser (marked by a red dashed box in Fig. 1) [12]. The inversion is simply controlled by the drive current in the pumping laser diode, thus yielding the convenient electrical tunability of $\mathrm{CR}$ central wavelength, achieved here without the changing of $\mathrm{CR}$ fiber. The $3 \mathrm{~dB}$ bandwidth of the emitted CR as a function of the input pump power is shown as the blue stars in Fig. 2(b). When the input $1035 \mathrm{~nm}$ pump power increases from 154 to
$308 \mathrm{~mW}$, the $3 \mathrm{~dB}$ bandwidth of CR increases from 14 to $36 \mathrm{~nm}$. The increase in CR bandwidth with increasing pump power can be ascribed to both SPM/cross-phase modulation effects and Raman-induced redshift of the pump pulses.

The emitted CR power as a function of the average input pump power is shown as the red circles in Fig. 2(b). The CR output shows a generation threshold at the pump power of around $130 \mathrm{~mW}$. The CR conversion efficiency grows significantly until the pump power reaches $180 \mathrm{~mW}$, after which the Cherenkov conversion efficiency saturates. We note here that the generated CR power is also dependent on the polarization state of the NL-3.0-850 [ $[6]$ and the highest CR output is generated by optimal rotational orientation of the PCF during the fiber splicing process. Figure 2(c) shows the far-field saturated VIS images of the CR emitted from our system, generated as the pump power was increasing in the range $150-300 \mathrm{~mW}$. These images exhibit the typical profile of the PCF mode [12].

In order to characterize the temporal profile of the generated CR pulses, an AC was measured. A $1 \mathrm{~mm}$ thick, $44.3^{\circ}$ cut beta barium borate (BBO) crystal was used in the autocorrelator for $\mathrm{CR}$. The $\mathrm{AC}$ of the $\mathrm{CR}$ with the output power of $1.7 \mathrm{~mW}$ is shown in Fig. 3 along with the $\mathrm{AC}$ of the pump pulse, measured at the end of the HCPCF laser compressor (i.e., before entering the CRgeneration fiber link). The FWHM of the AC trace of the $1035 \mathrm{~nm}$ pump pulse is $832 \mathrm{fs}$, whereas the FWHM of the $\mathrm{AC}$ trace for the generated $\mathrm{CR}$ pulse is $160 \mathrm{fs}$, i.e., more than five times shorter, which results from the nonlinear pump pulse compression in the CR fiber link. We note that the actual duration of the CR pulse is obviously even shorter than its AC FWHM value. The CR signals emitted in the output power range $1-2.5 \mathrm{~mW}$ had the AC FWHM in the range 145-225 fs. From the simulations $[\underline{13}, 14]$ we can estimate the pulse duration of the CR output generated from $10 \mathrm{~cm} \mathrm{NL-3.0-850} \mathrm{to} \mathrm{be} \mathrm{of} \mathrm{the} \mathrm{order} \mathrm{of} \mathrm{hundreds} \mathrm{of}$ femtoseconds, which matches our experimental results. The output power range 1-2.5 $\mathrm{mW}$ corresponds to pulse energies of 37-93 pJ. Such energies are rather high and are comparable to those used in previously published two-photon fluorescence microscopy experiments using
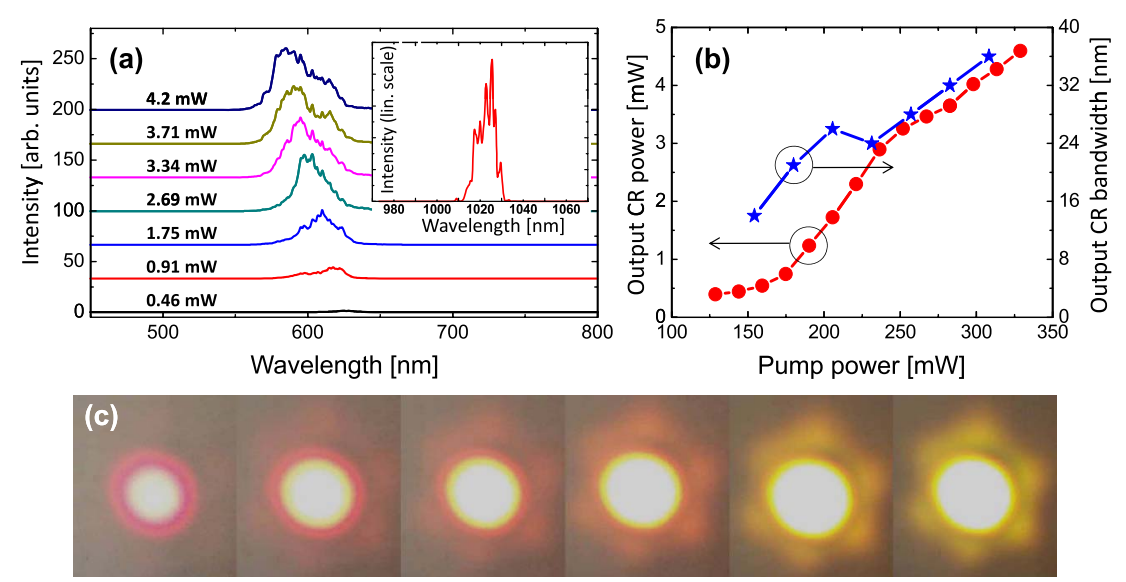

Fig. 2. (Color online) (a) Spectra of CR at variable output power. (Inset) Spectrum of the input femtosecond pump signal. (b) Generated CR output power (red circles) and $3 \mathrm{~dB}$ bandwidth (blue stars) as a function of increasing pump power. (c) (From left to right) Far-field saturated VIS images of the generated Cherenkov output as the pump laser power increases from 150 to $300 \mathrm{~mW}$. 


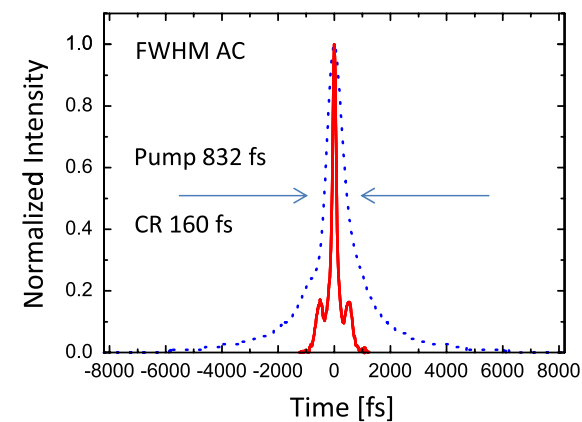

Fig. 3. (Color online) AC of the generated CR pulse (red solid) with the output power of $1.7 \mathrm{~mW}$, and the input pump pulse (blue dotted).

a filtered fiber $\mathrm{SC}[15,16]$. When the CR power increases from around 3 to $4 . \overline{\mathrm{mW}}$, the output CR power is found to temporarily degrade and then recover after about a minute, not leading to any permanent damage in the system. The experiments aimed at understanding this phenomenon are currently in progress.

In this Letter, we have shown a highly stable femtosecond CR source with convenient electrical tunability in the range $580-630 \mathrm{~nm}$ and multimilliwatt output powers based on monolithic all-fiber technology. By selecting a PCF with a smaller ZDW, similar tunability in the VIS and even UV spectral ranges can be realized $[6,17]$. As an example of stable operation, the CR output shown in Fig. 3 remains unchanged during the more than one month long operation of our system, during which over 200 on-off cycles for the whole system occurred. We believe that such stability of our system makes it promising for practical biophotonics applications, e.g., ultrafast spectroscopy, fluorescence lifetime imaging, and multiphoton microscopy, performed in the out-of-the-lab environments.

We acknowledge the Danish Council for Independent Research-Technology and Production Sciences (FTP) and the National Institutes of Health (grant 1 R01 EB012479) for financial support; B. Zhou, G. Villanueva, J. Thøgersen, and S. R. Keiding for valuable assistance; and NKT Photonics A/S for providing the PCFs.

\section{References}

1. B. M. Bolotovskii and V. L. Ginzburg, Sov. Phys. Usp. 15, 184 (1972).

2. P. K. A. Wai, C. R. Menyuk, Y. C. Lee, and H. H. Chen, Opt. Lett. 11, 464 (1986).

3. N. Akhmediev and M. Karlsson, Phys. Rev. A 51, 2602 (1995).

4. J. M. Dudley, G. Genty, and S. Coen, Rev. Mod. Phys. 78, 1135 (2006).

5. U. Møller, S. T. Sørensen, C. Jakobsen, J. Johansen, P. M. Moselund, C. L. Thomsen, and O. Bang, Opt. Express 20, 2851 (2012).

6. H. Tu and S. A. Boppart, Opt. Express 17, 9858 (2009).

7. H. Tu and S. A. Boppart, Proc. SPIE 7569, 75692D (2010).

8. X. Liu, J. Lægsgaard, and D. Turchinovich, Opt. Lett. 35, 913 (2010).

9. X. Liu, J. Lægsgaard, and D. Turchinovich, Opt. Express 18, 15475 (2010).

10. X. Liu, J. Lægsgaard, and D. Turchinovich, "Monolithic highly-stable Yb-doped femtosecond fiber lasers for applications in practical biophotonics," IEEE J. Sel. Top. Quantum Electron., doi:10.1109/JSTQE.2012.2183580 (to be published).

11. G. Chang, L. J. Chen, and F. X. Kärtner, Opt. Lett. 35, 2361 (2010).

12. D. Turchinovich, X. Liu, and J. Lægsgaard, Opt. Express 16, 14004 (2008).

13. I. Cristiani, R. Tediosi, L. Tartara, and V. Degiorgio, Opt. Express 12, 124 (2004).

14. D. R. Austin, C. M. de Sterke, B. J. Eggleton, and T. G. Brown, Opt. Express 14, 11997 (2006).

15. D. Li, W. Zheng, and J. Y. Qu, Opt. Lett. 34, 202 (2009).

16. J. Palero, V. Boer, J. Vijverberg, H. Gerritsen, and H. J. C. M. Sterenborg, Opt. Express 13, 5363 (2005).

17. N. Y. Joly, J. Nold, W. Chang, P. Hölzer, A. Nazarkin, G. K. L. Wong, F. Biancalana, and P. St. J. Russell, Phys. Rev. Lett. 106, 203901 (2011). 